\title{
Evaluation of teaching quality based on binary tree support vector machine
}

\author{
Xinghui $\mathrm{Wu}^{1,}{ }^{*}$, Zaifeng $\mathrm{Shi}^{2}$, Haihua Xing ${ }^{1}$, and Yisheng Xue ${ }^{1}$ \\ ${ }^{1}$ School of information science and technology, Hainan Normal University, Hainan Haikou \\ ${ }^{2}$ School of Chemistry and Chemical Engineering, Hainan Normal University, Hainan Haikou
}

\begin{abstract}
In order to solve the reliability of the evaluation results of teaching quality in universities and colleges, an improved model of teaching evaluation based on the Support vector machine was put forward. In this model, the evaluator does not need to give an evaluation result of the teacher's teaching quality, but gives the score of each evaluation index, and then calls the Support vector machine, automatic classification of teachers' teaching quality. The experiment proves that the improved algorithm can improve the teaching quality evaluation accuracy and the result is better.
\end{abstract}

Keywords: Fuzzy, Binary tree, Support vector machine, Teaching quality.

\section{Introduction}

Teaching quality evaluation is a multi-objective and multi-level optimization work, which is a guarantee system for improving teaching quality and cultivating talents ${ }^{[1-3]}$. But there are many ways and contents of evaluation, and there is a complicated non-linear mapping relationship between evaluation index and teaching quality ${ }^{[4]}$.

Currently, the commonly used evaluation methods of teaching quality in universities colleges mainly include: Multiple Linear Regression, grey correlation, analytic hierarchy and process, neural network, fuzzy clustering analysis and Support vector machine, etc $\left.{ }^{[5-6}\right]$. With strong ability of classification, neural network can improve the teaching quality accuracy, but in the application process, due to its limitation and easy falling into the extreme point, the result will be inconsistent with the actual result, and inefficient. Support Vector machine is a kind of machine learning method with small sample and strong nonlinear approximation ability, but in the evaluation of class teaching quality in universities and colleges, the evaluation accuracy is closely related to the parameters selected. To obtain high accuracy evaluation results, the evaluation model parameters need to be optimized, the optimal Support vector machine parameters is necessary for improving the evaluation results ${ }^{[7]}$.

To improve the evaluation accuracy of class teaching quality in Universities and Colleges, concerned with the method of evaluation, an improved teaching evaluation model

* Corresponding author: 793957623@qqq.com 
of fuzzy binary tree support vector machine is proposed to evaluate classroom teaching. The model algorithm is based on the binary tree support vector machine classification algorithm to establish the fuzzy membership function, through the KNN fuzzy membership measurement method to calculate different penalty values. The results show that the evaluation accuracy can be improved with the modified algorithm, and better evaluation results are got.

\section{Fuzzy binary tree support vector machine}

\subsection{Fuzzy support vector machine}

When there are no outliers or noises in the data set, the classification accuracy of SVM is better. In practice, if there are outliers or noises in the training samples, the classification accuracy of SVM will be affected. In this case, Lin, a Taiwan scholar, proposed fuzzy support vector machine (FSVM) ${ }^{[8]}$. The design principle of FSVM is aimed at the influence of noise and isolation on the classification performance of support vector machine. Fuzzy parameters are introduced into support vector machine. Different training samples are given different values, and isolated points and noise are given smaller values, so as to achieve their influence on classification accuracy and precision ${ }^{[9]}$.

Let the training set be expressed as $\left\{\left(\mathrm{x}_{1}, \mathrm{y}_{1}, \mathrm{~s}_{1}\right), \ldots,\left(\mathrm{x}_{\mathrm{n}}, \mathrm{y}_{\mathrm{n}}, \mathrm{s}_{\mathrm{n}}\right)\right\}$, where $\mathrm{x}_{\mathrm{i}} \in \mathrm{R}^{\mathrm{n}}, \mathrm{y}_{\mathrm{i}} \in$ $\{1,-1\}, 0<s_{i} \leq 1, s_{i}$ is the fuzzy membership function, $I=1,2, \ldots$. L. for the linear problem, the optimal classification hyperplane is found to be the optimal solution of the following objective function ${ }^{[10]}$ :

$$
\begin{aligned}
& \min \frac{1}{2}\|\omega\|+C \sum_{i=1}^{l} s_{i} \delta_{i} \\
& \text { s.t. } \quad y_{i}\left(\omega^{T} x_{i}+b\right) \geq 1-\delta_{i}, i=1,2, \ldots, l \\
& \delta_{i} \geq 0, i=1,2, \ldots \ldots, l
\end{aligned}
$$

Introduce Lagrange function $\alpha_{\mathrm{i}}(\mathrm{i}=1,2, \ldots, \mathrm{l})$, The dual function is obtained as follows

$$
\begin{gathered}
\min \frac{1}{2} \sum_{i=1}^{l} \sum_{j=1}^{l} y_{i} y_{j} \alpha_{i} \alpha_{j}\left(x_{i} \cdot x_{j}\right)-\sum_{i=1}^{l} \alpha_{i} \\
\text { s.t. } y_{i} \alpha_{i}=0 \\
0 \leq \alpha_{i} \leq s_{i} C, i=1,2, \ldots . l
\end{gathered}
$$

For the nonlinear solution, the kernel function is introduced $\mathrm{K}\left(\mathrm{x}_{\mathrm{i}} \cdot \mathrm{x}_{\mathrm{j}}\right)$, Then the dual classification problem of quadratic programming can be expressed as follows:

$$
\min \frac{1}{2} \sum_{i=1}^{l} \sum_{j=1}^{l} y_{i} y_{j} \alpha_{i} \alpha_{j} K\left(x_{i} \cdot x_{j}\right)-\sum_{i=1}^{l} \alpha_{i}
$$

The fuzzy optimal classification function is obtained by solving the above problems:

$$
\begin{aligned}
\mathrm{f}(\mathrm{x})= & \operatorname{sgn}\left\{\sum_{i=1}^{l} \alpha_{i}^{*} y_{i} \mathrm{~K}\left(x_{i} \cdot x_{j}\right)+b^{*}\right\} \\
& \text { among: } \mathrm{b}^{*}=y_{i}-\sum_{i=1}^{l} y_{i} \alpha_{i} K\left(x_{i} \cdot x_{j}\right)
\end{aligned}
$$




\subsection{Binary tree support vector machine}

The binary tree Support vector machine classification Algorithm (BT-SVMs) divides classes into 2 classes, then subclasses are further divided into two subclasses, and so on, until all nodes contain only a single class, then a binary SVM classifier is trained at each non-leaf node. When the binary tree structure is near to general binary tree, the optimal classification accuracy and training speed can be obtained. For Class K problems, only k-1 binary classifiers needs to be constructed by BTSVM, but there are also two important problems: first, the structure of binary tree is uncertain, there will be many different kinds of binary tree structures for the same one multi class classification problem, different structures will lead to different models. Figure 1 show two binary tree structures used commonly in a four-class problem. Secondly, this method will lead to the phenomenon of "error accumulation" [7], that is, at the upper node while the classification is mistaken, the passed error will lead the subsequent nodes to lose the classification meaning, so, the BTSVM upper node's sub-classifier greatly impact on the whole classifier performance. To get the optimal classification result, a better binary tree structure must be constructed according to the real situation.
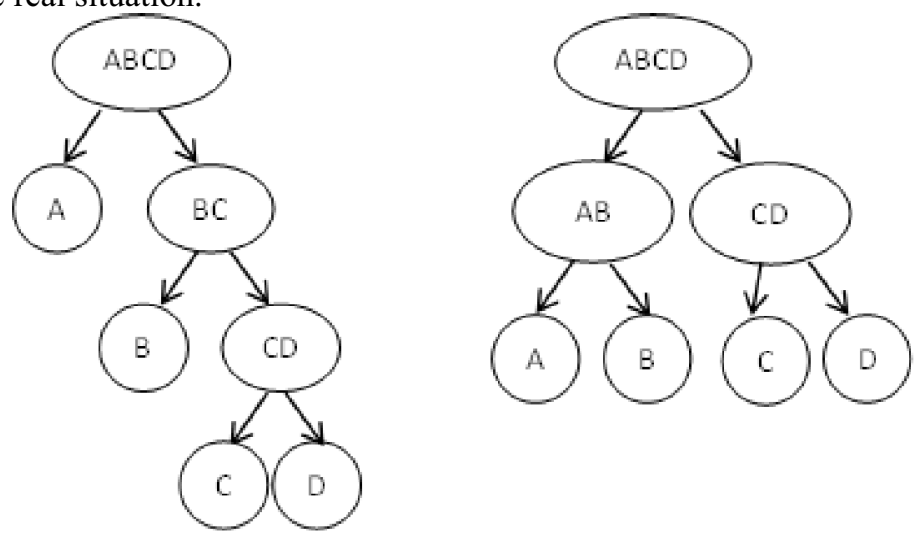

Fig. 1. Common binary classification tree structure.

\subsection{Fuzzy binary tree support vector machine}

The fuzzy binary tree Support vector machine (BT-FSVMs) algorithm is a combination of modular Support vector machine and Support vector machine, the Algorithm first constructs the appropriate tree structure according to the training samples according to the construction method of the Support vector machine tree structure, and then carries on the mold processing to the samples at each node, finally, the decision function is obtained by using the fuzzy Support vector machine. BT-FSVMs can reduce the influence of noise and outliers, and at the same time, reduce the influence of "error accumulation" of Support vector machine algorithm on classification accuracy, improved the classification accuracy of binary tree Support vector machine.

\subsection{Algorithm of BT FSVMs fuzzy membership function}

In the Support vector machine, the membership function chosen, may have a greater impact on the accuracy of the Algorithm. In this paper, the Correlation Coefficient is introduced into the selection of membership function, and the method of taking the absolute value of the correlation Coefficient between sample and cluster center as membership function is put 
forward, in order to realize the method of constructing the tree structure and determining the membership function based on the Correlation Coefficient, a smaller value is given ${ }^{[12]}$. This paper adopts the KNN-based method for determining the Modulus of fire and the degree of membership.

\section{Teaching quality evaluation model based on BT FSVMs}

\subsection{Teaching quality evaluation index}

Through the analysis of the factors affecting the teaching quality and the results of the degree of influence on the teaching quality, a variety of indicators of teaching quality evaluation are determined. This paper constructs the evaluation index system of college classroom teaching quality from the aspects of teaching attitude, teaching content, teaching method and teaching effect, as shown in Table 1.

Table 1. Classroom teaching quality index.

\begin{tabular}{|c|c|c|c|}
\hline Classification & Content & $\begin{array}{l}\text { Weight } \\
\text { coefficient }\end{array}$ & $\begin{array}{l}\text { Weight } \\
\text { coefficient }\end{array}$ \\
\hline \multirow{3}{*}{$\begin{array}{l}\text { Teaching } \\
\text { attitude }\end{array}$} & X1: Class earnest, full of spirit & \multirow{3}{*}{0.35} & 0.35 \\
\hline & $\begin{array}{l}\text { X2: Go to and from class on time and do not use } \\
\text { mobile phones during the teaching process; Mediation } \\
\text { course less }\end{array}$ & & 0.42 \\
\hline & $\begin{array}{l}\text { X3: Care for students, both in and out of class are } \\
\text { happy to answer problems }\end{array}$ & & 0.23 \\
\hline \multirow{3}{*}{$\begin{array}{l}\text { Content of } \\
\text { courses }\end{array}$} & X4: Skillful and well-organized & \multirow{3}{*}{0.25} & 0.34 \\
\hline & $\begin{array}{l}\text { X5: The key is prominent, the detail, difficulty and } \\
\text { depth are appropriate }\end{array}$ & & 0.38 \\
\hline & $\begin{array}{l}\text { X6: Theory and content reflect the progresses of the } \\
\text { subject }\end{array}$ & & 0.28 \\
\hline \multirow{3}{*}{$\begin{array}{l}\text { Teaching } \\
\text { method }\end{array}$} & $\begin{array}{l}\mathrm{X} 7 \text { : The teaching method is flexible and diverse, can } \\
\text { use multimedia appropriately, and suits the teaching } \\
\text { content }\end{array}$ & \multirow{3}{*}{0.25} & 0.42 \\
\hline & $\begin{array}{l}\text { X8: The lecture is full of inspiration and easy to learn, } \\
\text { and talk with students any time. }\end{array}$ & & 0.22 \\
\hline & $\begin{array}{l}\text { X9: Reasonable assignment, and effective review and } \\
\text { feedback }\end{array}$ & & 0.36 \\
\hline \multirow{3}{*}{$\begin{array}{l}\text { Teaching } \\
\text { efficiency }\end{array}$} & $\begin{array}{l}\text { X10: Students have high enthusiasm in class and good } \\
\text { classroom atmosphere }\end{array}$ & \multirow{3}{*}{0.15} & 0.32 \\
\hline & X11: Students got the knowledge in the course & & 0.36 \\
\hline & $\begin{array}{l}\text { X12: Students' learning interest and ability are } \\
\text { improved in the course }\end{array}$ & & 0.32 \\
\hline
\end{tabular}

\subsection{Flow chart of teaching quality evaluation process based on BT FSVMs}

Combined with the above teaching quality evaluation index, this paper puts forward a teaching quality evaluation model based on Support vector machine. In order to improve the efficiency and accuracy of teaching evaluation, the model combines Support vector machine and Support vector machine to solve the problem of multi-classification in teaching quality evaluation. Figure 2 shows a workflow diagram for the evaluation of the quality of college classes based on the Support vector machine. 


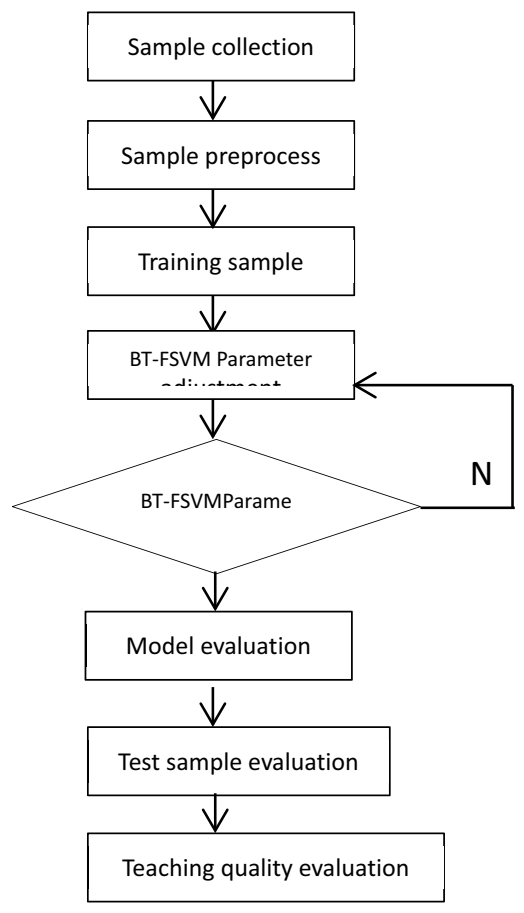

Fig. 2. Flow chart of teaching quality evaluation.

\section{Evaluation experiment Process Based on BT FSVMs}

\subsection{Experimental data set}

The data of this paper comes from the teaching quality evaluation data of a university in Hainan, with a total of 180 data samples. The data is divided into training samples and test samples. Because the evaluation index units of teaching quality are different, and the range of data change is different, it is necessary to preprocess them. The preprocessing needs to normalize the final data so that the input data is between $[0,1]$. Normalization uses the following formula:

$$
\mathrm{x}_{i}^{*}=\frac{x_{i}-x_{\min }}{x_{\max }-x_{\min }}
$$

where $x_{i}^{*}, x_{i}$ is the value before and after normalization, $x_{\max }, x_{\min }$ represents the maximum and minimum values in the sample.

The first 140 data are used as training samples, and the last 40 data are used as test samples. The normalized learning samples are input into the fuzzy binary tree support vector machine classifier for learning. The fuzzy binary tree support vector machine is trained by using the KNN based fuzzy membership measurement method described above, and the sequential minimum optimization algorithm (SMO) is used to solve the convex quadratic programming problem of fuzzy support vector machine. Smo is an optimization method in support vector machine. In this experiment, the kernel function of SVM is Gaussian radial basis function, and the parameter $\mathrm{K}\left(\mathrm{x}_{\mathrm{i}}, \mathrm{x}_{\mathrm{j}}\right)=\exp \left(\frac{-\gamma\left\|\mathrm{x}_{\mathrm{i}}-\mathrm{x}_{\mathrm{j}}\right\|^{2}}{2 \gamma^{2}}\right)$, the penalty parameter $\mathrm{C}$ and the kernel function parameter $\gamma$ The grid search method is used to select, 
and the 10 fold cross validation is used to find the optimal combination of these parameters, and $\mathrm{C}$ and $\mathrm{C}$ are determined $\gamma$ The range is $\left(2^{-5}, 2^{10}\right)$. The experimental parameters are used to train the training samples. The test samples are classified, and the fuzzy binary tree support vector machine classifier is used to classify the test samples. Finally, the results of teaching quality evaluation are output.

\subsection{Results and evaluation}

In order to evaluate the model, BT-FSVMs is tested with FSVM and BT-SVMs respectively, and the accuracy and time consumption of the three algorithms are obtained. The results show that the prediction accuracy of BT-FSVMs Algorithm is higher than FSVM, BT-SVMs Algorithm. The FSVM Algorithm takes a longer time, but the time difference between BT-FSVMs and BT-SVMs Algorithm is very small. The results of the experiment are shown in Table 2.

Table 2. Comparison of evaluation accuracy.

\begin{tabular}{|l|l|l|l|}
\hline Algorithm & Accuracy (\%) & Training time (ms) & Test time (ms) \\
\hline BT-FSVMs & 96.68 & 21.54 & 5.32 \\
\hline BT-SVMs & 94.46 & 24.43 & 6.45 \\
\hline FSVM & 93.38 & 25.56 & 8.56 \\
\hline
\end{tabular}

\section{Conclusions}

Classroom teaching plays an important role in the process of teaching. Scientific evaluation of classroom teaching quality is needed to improve of teaching and education. To evaluate the classroom teaching quality, the indexes involve teachers' teaching ability, attitudes, methods, contents, teaching effect and learning effect etc. In order to improve the evaluation accuracy and efficiency, this paper presents an evaluation model based on SVM. In this model, the evaluator does not need to give an evaluation result of the teacher's teaching quality, but gives the score of each evaluation index, and then calls the Support vector machine, this algorithm designs an evaluation model which accords with the teaching quality of Modern University classroom education. The data are simulated and the simulation results are analyzed. Results indicate that the evaluation model of teaching quality based on the modified Algorithm gives higher time efficiency and accuracy in teacher classification, and improves the efficiency of evaluation.

Thanks are given to Education Department and Science \& Technology Department of Hainan Province for funding the studies of this paper (Hnjg2020-31, Hnjg2020-32, QJY20181071, 2019RC182).

\section{References}

1. Hu Shuchang, Zhao Xiaoqin, Liu Zhenyou. Research and practice of evaluation index system of teachers' classroom teaching quality. Journal of Gannan Medical College, 2001, 1: 76-79.

2. Wu Enying, Lv Jia. Research on multiclass classification algorithm based on binary tree SVM. Journal of Chongqing Normal University (Natural Science Edition), 2016, 33(3): 103-106.

3. Li Yanling, Su Yidan. Application of improved binary tree support vector machine in multi-classification. Computer Technology and Development, 2014, 7: 181-184. 
4. Li Yanling. Application of BT-SVM multi-classification algorithm in teaching quality evaluation. Thesis for master degree: Guangxi University, P.R.China, 2014.

5. Cheong S. Support vector machines with binary tree architecture for multi -class classification. Neural Information Processing-Letters and Reviews, 2004, 2(3): 47-51.

6. Fan Baichao, Wang Jianyu, Bo Yuming. Binary tree SVM multi-class classification algorithm with feature selection. Computer Engineering and Design, 2010,31(12): 2823-2825.

7. Hsu Chih-Wei, Lin Chih-Jen. A comparison of methods for multi-class support vector machines. IEEE Transactions on Neural Networks, 2002, 13(2): 415-425.

8. Zhao Liang. An improved SVM based multi-class classification algorithm. Computer Applications and Software, 2014, 31(12): 233-236.

9. Xia Siyu, Pan Hong, Jin Lizuo Multi-class SVM method based on non-balanced binary tree. Computer Engineering and Applications, 2009, 45(17): 167-169.

10. Song Zhaoqing, Chen Yao. An overview of multi-class algorithm based on support vector machine. Journal of Naval Aeronautical and Astronautical University. 2015, 30(5): 442-446.

11. Chen Lijie. Teaching quality evaluation model based on neural network. Vocational Education Research, 2005(7): 150-151.

12. Zhou Shiguan, Su Rina. Application of fuzzy neural network in the quality evaluation of classroom teaching. Computer Simulation, 2008, 25(5): 287-289. 